\title{
Molecular Diagnosis of Babesia canis Infection with Concurrent of Evans Syndrome in a Labrador Dog
}

\author{
M. Saravanan*, P. Selvaraj, S. Yogeshpriya, M. Venkatesan, \\ P.K. Ramkumar and K. Kannan
}

\author{
Veterinary Clinical Complex, Veterinary College and Research Institute, Orathanadu, \\ Thanjavur - 614 625, TamilNadu Veterinary and Animal \\ Sciences University (TANUVAS), India \\ *Corresponding author
}

\section{A B S T R A C T}

\section{Keywords}

Babesia canis, IMTP, IMHA, Flow cytometry, Evans syndrome

Article Info

Accepted:

17 November 2019 Available Online:

10 December 2019
Clinical case is five years old female Labrador dog presented to Small Animal Medicine unit of TVCC, VCRI, Orathanadu. History is dullness and anorexia two days. Recurrent tick infestations, anemic and thrombocytopenic crisis were regularly. Vaccination and deworming were regularly. Physical examination revealed dullness, pale mucosa, vital parameters were normal and ticks on interdigital space and in ears were noticed. Distended abdomen, hepatosplenomegaly and petechial hemorrhage on ventral abdomen were observed. Hematology revealed anemia and thrombocytopenia. Negative for blood born parasites in peripheral blood smear. Serum biochemical and urine analysis found to be normal. Serum and EDTA blood were submitted for molecular testing. Flow cytometry revealed Immune-mediated hemolytic anemia (IMHA) and Immune-mediated thrombocytopenia (IMTP). MAT negative for leptospira. Babesia canis positive by PCR method at 394bp. Haematology alteration was studied before and after therapy. Ultrasonography revealed hepatosplenomegaly. Based on PCR and flow cytometry the case was confirmed Evans syndrome with Concurrent Babesia canis in a Labrador dog. Dog treated with Inj. Diminazene aceturate @ $3.5 \mathrm{mg} / \mathrm{kg}$ IM single dose, Inj. Ringers lactate@100 ml IV, Inj. Ascorbic acid @ 5 ml IV, Inj. Vitamin B complex @ 1ml IV, Inj. Prednisone @ 0.5mg/kg IM, Tab. Doxycycline @ 10mg/kg PO and Syrup. Thrombup @ $15 \mathrm{ml}$ BID PO. Dog showed clinical improvement as well as hematology parameters.

\section{Introduction}

Babesiosis is one among the deadly tick-borne hemaoprotozone infection in the canines. Causative agents of canine babesiosis are Babesia canis, B. gibsoni, and B. vogeli, intracellular protozoan parasites of canine erythrocytes (Breitschwert, 1984). Babesia infection causes autoimmune disorder like Immune-mediated haemolytic anaemia (IMHA) and immune mediated thrombocytopenia (ITP) in dogs and it may occur individually or concurrently. If they occur together they call it as Evans syndrome 
(Norkus, 2012). Evans syndrome with Babesia in canines leads destruction of RBCs, platelets, oxidative stress and endothelial injury (Wingfield and Raffe, 2002; Di Cicco and Birkenheuer, 2012).

\section{Case report}

Five years old female Labrador was brought to the Veterinary Clinical Complex, Veterinary College and Research Institute, Orathanadu with history of dullness and anorexia for the past two days. Recurrent tick infestations, anemic and thrombocytopenic crisis were reported 6 month back. Vaccination and deworming were regularly done. On general clinical examination revealed dull and depressed, Woebegone, pale mucus membrane and HR-102/minute, RR$45 /$ minute and rectal temperature $38.9^{\circ} \mathrm{C}$. On physical examination revealed distended abdomen, hepatosplenomegaly, Petechial hemorrhage on ventral abdomen, and ticks noticed on ear and interdigital space.

Samples collected: EDTA blood - CBC by Auto analyzer, Peripheral blood smear Blood protozoal infection, Urinalysis, Ticks for identification. CBC revealed anemia and thrombocytopenia. No blood parasite could be detected through peripheral blood smear. No abnormalities could be detected in urinalysis. Rhipicephalus sanguineous tick was identification.

Since the animal was with intermittent tick infestation and anemia - Serum \& EDTA blood were collected and submitted for molecular testing to Zoonoses Research Lab and TRPVB Madhavaram, TANUVAS, Chennai for the diagnosis of Leptospirosis, Blood protozoal infection (Babesiosis, Ehrlichiosis, Trypanosomiasis and Hepatozoonosis) and autoimmune diseases (IMHA \& IMTP) condition flow cytometry test for anti-platelets and anti-red blood cells antibodies were performed. Microscopic agglutination test (MAT) was negative for Leptospira sp. PCR was positive for Babesia canis at 394bp. IgM-anti-platelets and IgGanti-red blood cells antibodies were detected and it confirms IMHA and IMTP (Figure 1 and Table 1). Based on the results of PCR and flow cytometry the case was confirmed as Babesia canis infection with concurrent of Evans syndrome in a Labrador dog.

Ultrasonographic examination of the abdomen revealed hepatosplenomegaly. Treatment initiated with Inj. Diminazene aceturate @ 3.5mg/kg IM single dose, Tab. Doxycycline @10mg/kg SID PO for 15 days, Tab. Prednisolone started @ 0.5mg/kg PO for 30 days, @ 0.25mg/kg PO for 15 days followed by alternate days as tapering. Supportive with syrup. Thrombup @ $15 \mathrm{ml}$ BID PO for 30 days and syrup. Astymin @ $15 \mathrm{ml}$ BID PO for 30 days. Haematology alteration was studied before and after therapy. Animal showed clinical improvement like alertness, food intake and improvement in hematology values were also noticed (Table 2).

Table.1 Flow cytometry of Babesia canis dog with IMHA and IMTP

\begin{tabular}{|l|c|c|}
\hline & IMHA & IMTP \\
\hline Unstained & 1.46 & 2.07 \\
\hline IgG & 1023.94 & 10688.84 \\
\hline IgM & 607.82 & 3022.33 \\
\hline
\end{tabular}


Table. 2 Changes of blood values in Babesia canis dog with IMHA and IMTP

\begin{tabular}{|l|c|c|c|c|}
\hline Parameter & Day 1 & Day 4 & Day 30 & Day 45 \\
\hline Hb $(\mathbf{g} / \mathbf{d l})$ & 5.9 & 6.4 & 8.6 & 8.8 \\
\hline PCV $(\mathbf{\%})$ & 17.63 & 19.70 & 25.16 & 26.76 \\
\hline RBC $(\times \mathbf{1 0} / \boldsymbol{\mu L})$ & 2.70 & 2.99 & 4.05 & 4.30 \\
\hline $\mathbf{W B C}(\times \mathbf{1 0} / \boldsymbol{\mu L})$ & 6.75 & 6.56 & 8.99 & 7.68 \\
\hline PT $(\times \mathbf{1 0} / \boldsymbol{4} \mathbf{L})$ & 8.1 & 12.1 & 15.9 & 22.4 \\
\hline
\end{tabular}

Figure.1 Flowcytometric evaluation of anti-platelets and anti-red blood cells antibodies

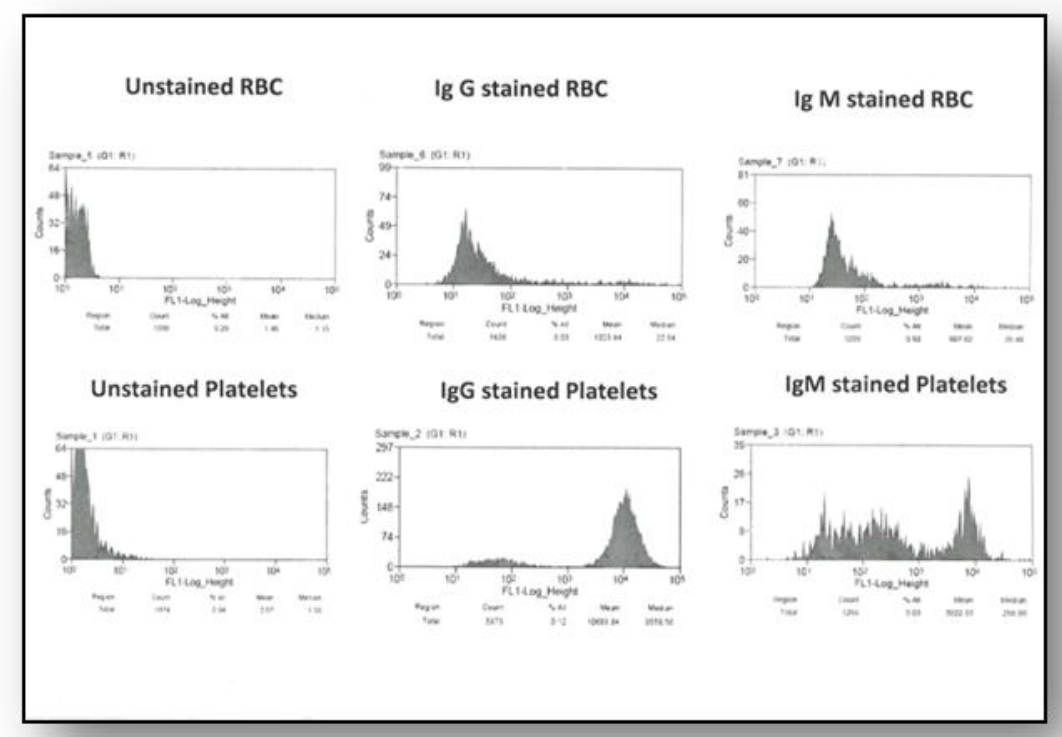

B. canis is a large intraerythrocyte piroplasm which causes hemolytic crisis and Immunemediated hemolytic anemia (IMHA) occur secondary to the inappropriate production of antierythrocyte membrane antibodies (Archer and Mackin, 2013). Nalubamba et al., (2015) recorded babesiosis in forty-five percent of the dogs were infested with ticks mainly of the genus Rhipicephalus. Thrombocytopenia and hematologic abnormalities were occurs in babesiosis as alone or together and mostly the bleeding disorder or coagulopathy are associated with DIC and immune-mediated platelet destruction (Tuttle et al., 2003; Taboada and Lobetti, 2006).
The common clinical signs of babesiosis are anorexia, fever, depression, lethargy, pale mucosae, splenomegaly, poor body condition, and thrombocytopenia (Taboada and Lobetti, 2006). Sarma et al., (2016) observed in his study hepato-splenomegaly in dogs naturally infected babesiosis by ultrasonographically.

PCR assay is the high specificity and sensitivity test to confirm the Babesia spp infection in dogs (Di Cicco and Birkenheuer, 2012) by detecting low levels of parasitemia, recognizing subclinical infections and monitoring response to therapy (Boozer and Macintire, 2015). It correlated with the 
present cases $B$. canis was positive in PCR assay and negative in peripheral blood smear.

Flow cytometry are considered to be more rapid, cost-effective, sensitive methods to determine erythrocytes-bound immunoglobulins (Quigley et al., 2001) and Platelet bound immunoglobulins (Dircks et al., 2009). Norton and Roberts (2006) reported Evans syndrome is a pathological condition, defined by the combination (either simultaneously or sequentially) of IMT and IMHA in the absence of known underlying etiology. Evans syndrome have been documented in dogs by Michimoto et al., (2004) , Goggs et al., (2008) and Giannuzz et al., (2014).

Treatment given in the present case was correlated with (Ayoob et al., 2010; Sarma et al., 2014). Diminazene aceturate or Imidocarb dipropionate are very much effective for the elimination of Babesia canis from the host (Schoeman, 2009) and in the present case Diminazene aceturate @ $3.5 \mathrm{mg} / \mathrm{kg}$ IM showed better recovery. Prednisolone is a one of the potent immuno suppressive drug that reduce phagocytosis of RBCs, decrease production of cytokines and immunoglobulin IgG and which is commonly used in autoimmune disorders like IMHA and IMTP in dogs (Breton, 2015)

In conclusions, etiology based treatment along with adjuvant therapy is always helps in early and complete recovery. Molecular diagnostic tool like PCR is essential to rule out tick born infection in dogs those which are intermittent or persistent tick infestation and even though negative in the routine peripheral blood smear examination. Persistent subclinical tick born infection may prone for the development of Immune mediated diseases like IMHA and IMTP. Flow cytometry helped in the diagnosis of IMHA and IMTP in dogs.

\section{Acknowledgement}

The Authors are grateful to The Director of Clinics TANUVAS Chennai and Professor and Head VCC VCRI, Orathanadu to provide all the facilities for carrying out this study.

\section{References}

Archer, T and Mackin, A. 2013. Diagnosis of Immune-Mediated Hemolytic Anemia. Today's Veterinary Practice. 32-36.

Ayoob, A.L., Hackner, S.G and Prittie, J. 2010. Clinical management of canine Babesiosis - clinical practice review. Journal of Veterinary Emergency and Critical Care. 20(1): 77-89.

Boozer, L and Macintire, D. 2005. Babesia gibsoni: An Emerging Pathogen in Dogs. Compendium. 33-42.

Breitschwert, E. 1984. Babesiosis. Clinical Microbiology and Infectious Diseases of the Dog and Cat. Philadelphia: WB Saunders. Pp 796-805.

Breton, A. 2015. Evans Syndrome: Breaking down IMHA and ITP. NZVNJ.,75 (21): $24-28$

Di Cicco, M.F and Birkenheuer, A.J. 2012. Canine Babesiosis - Peer Reviewed. NAVC Clinician's Brief. July 31-35.

Dircks, B.H., Schuberth, H.J and R. Mischke, R. 2009. Underlying diseases and clinicopathologic variables of thrombocytopenic dogs with and without platelet-bound antibodies detected by use of a flow cytometric assay: 83 cases (2004-2006)," Journal of the American Veterinary Medical Association. 235(8): 960-966.

Giannuzzi, A.P., De Simone, A., Ricciardi, M and Gernone, F. 2014. Presumptive Ischemic Brain Infarction in a Dog with Evans' Syndrome - Case Report. Case Reports in Veterinary Medicine. http://dx.doi.org/10.1155/2014/456524 
Goggs, R., Boag, A.K and Chan, D.L. 2008. Concurrent immune-mediated haemolytic anaemia and severe thrombocytopenia in 21 dogs. Veterinary Record. 163(11): 323-327.

Michimoto, T., Okamura, T., Suzuki, K., Watari, T., Kano, R and Hasegawa, A. 2004. Thiazole orange positive platelets in a dog with Evans' syndrome. Journal of Veterinary Medical Science. 66(10): 1305-1306.

Nalubamba, K.S., BasimbiMudenda, N., Namwila, M., SusanMulenga, C., Bwalya, E.C., M'kandawire, E., Saasa, N. Hankanga, C., Oparaocha, E and Simuunza, M. 2015. A Study of Naturally Acquired Canine Babesiosis Caused by Single and Mixed Babesia Species in Zambia: Clinicopathological Findings and Case Management. Journal of Parasitology Research. http://dx.doi.org/10.1155/2015/985015

Norkus, C. 2012. Veterinary Technician's Manual for Small Animal Emergency and Critical Care", Chapter 11: Specific Organ System Disorders, ed. Campbell M., Ames, Iowa, WileyBlackwell. Pp 243-252.

Norton, A and Roberts, I. 2006. Management of Evans syndrome. British Journal of Haematology. 132(2): 125-137.

Quigley, K.A., Chelack, B.J., Haines, D.M and Jackson, M.L. 2001. Application of a direct flow cytometric erythrocyte immunofluorescence assay in dogs with immune-mediated hemolytic anemia and comparison to the direct antiglobulin test. Journal of Veterinary Diagnostic Investigation. 13(4): 297-300.

Sarma, K., Mondal, D.B and Saravanan, M. 2016. Ultrasonographic changes in dogs naturally infected with tick borne intracellular diseases. J Parasit Dis., 40(2): 248-251.

Sarma, K., Saravanan, M., Kumar, A., Kamili, N.M., Mahandran, K., Mondal, D.B and Dey, S. 2014. Concomitant Babesia gibsoni and Ehrlichia canis infection in a dog with poly systemic dysfunctions and its therapeutic management - A case report. Indian J. Vet. Med. 35(1): 7477.

Schoeman, J.P. 2009. Canine babesiosis. Onderstepoort J Vet Res., 76(1):5966.

Taboada, J and Lobetti, R. 2006. Babesiosis, In: Greene C. ed. Infectious Diseases of the Dog and Cat, $3^{\text {rd }}$ edn. St Louis: WB Saunders Co. Pp 722-735.

Tuttle, A.D., Birkenheuer, A.J., Juopperi, T., Levy, M.G and Breitschwerdt, E.B. 2003. Concurrent bartonellosis and babesiosis in a dog with persistent thrombocytopenia. J Am Vet Med Assoc., 223(9):1306-1310.

Wingfield, W., Raffe, M.. 2002. Veterinary ICU Book. Chapter 46: ImmuneMediated Hemolytic Anemia, ed. Faudskar L., Jackson, WY: Teton New Media. Pp: 710-720.

\section{How to cite this article:}

Saravanan, M., P. Selvaraj, S. Yogeshpriya, M. Venkatesan, P.K. Ramkumar and Kannan, K. 2019. Molecular Diagnosis of Babesia canis Infection with Concurrent of Evans Syndrome in a Labrador Dog. Int.J.Curr.Microbiol.App.Sci. 8(12): 2522-2526. doi: https://doi.org/10.20546/ijcmas.2019.812.295 